\title{
The Antifungal Mechanism of Amphotericin B Elucidated in Ergosterol and Cholesterol-Containing Membranes Using Neutron Reflectometry
}

\author{
Robin Delhom ${ }^{1,2,3}\left(\mathbb{D}\right.$, Andrew Nelson ${ }^{4}\left(\mathbb{D}\right.$, Valerie Laux ${ }^{1}$, Michael Haertlein ${ }^{1}$, \\ Wolfgang Knecht ${ }^{3,5}$, Giovanna Fragneto ${ }^{1}$ (D) and Hanna P. Wacklin-Knecht 2,6,*(D) \\ 1 Institut Laue-Langevin, 71 Avenue des Martyrs, CS 20156, 38042 Grenoble CEDEX 9, France; \\ robin.delhom@gmail.com (R.D.); laux@ill.fr (V.L.); haertlein@ill.fr (M.H.); fragneto@ill.fr (G.F.) \\ 2 European Spallation Source ERIC, P.O. Box 176, 22100 Lund, Sweden \\ 3 Department of Biology, Lund University, Sölvegatan 35, 22362 Lund, Sweden; wolfgang.knecht@biol.lu.se \\ 4 Australian Centre for Neutron Scattering, Australian Nuclear Science and Technology Organization, \\ Locked Bag 2001, Kirrawee DC, NSW 2232, Australia; andrew.nelson@ansto.gov.au \\ 5 Lund Protein Production Platform, Lund University, Sölvegatan 35, 22362 Lund, Sweden \\ 6 Department of Chemistry, Division of Physical Chemistry, Lund University, P.O. Box 124, \\ 22100 Lund, Sweden \\ * Correspondence: hanna.wacklin-knecht@ess.eu
}

Received: 10 November 2020; Accepted: 4 December 2020; Published: 6 December 2020

check for updates

\begin{abstract}
We have characterized and compared the structures of ergosterol- and cholesterol-containing 1-palmitoyl-2-oleoyl-sn-glycero-3-phosphocholine (POPC) membranes before and after interaction with the amphiphilic antifungal drug amphotericin $\mathrm{B}(\mathrm{AmB})$ using neutron reflection. AmB inserts into both pure POPC and sterol-containing membranes in the lipid chain region and does not significantly perturb the structure of pure POPC membranes. By selective per-deuteration of the lipids/sterols, we show that AmB extracts ergosterol but not cholesterol from the bilayers and inserts to a much higher degree in the cholesterol-containing membranes. Ergosterol extraction by AmB is accompanied by membrane thinning. Our results provide new insights into the mechanism and antifungal effect of AmB in these simple models of fungal and mammalian membranes and help understand the molecular origin of its selectivity and toxic side effects.
\end{abstract}

Keywords: amphotericin B; lipid membranes; POPC; ergosterol; cholesterol; neutron reflection

\section{Introduction}

Among clinically used antifungal treatments, amphotericin B (AmB), an amphiphilic macrocyclic polyene, is remarkable because of its broad spectrum of activity against different organisms, and only low frequency of resistance [1-3]. AmB has been used as a last resort treatment for life-threatening systemic fungal infections for over 50 years, but its mechanism at the molecular level is still unclear. It is accepted that AmB acts by preferentially binding to ergosterol [4,5], which is present in fungal cell membranes, over the cholesterol found in mammalian membranes [6,7], which forms the basis of its fungal specificity. However, whether or not this difference in affinity is related to differences in the mechanism of AmB binding to membranes containing different sterols is still an open question, as is the role and importance of the membrane phospholipid composition. This could be particularly important as AmB has a relatively high toxicity that is often dose-limiting in its clinical applications, and this has been proposed to be due to its interaction with cholesterol-containing human host cell membranes.

For many years, aqueous pore formation induced by AmB-sterol complexes was considered to be the main mechanism of its antifungal activity, as electrochemistry, fluorescence, and UV spectroscopy, 
surface plasmon resonance, NMR, and molecular dynamics showed consistent evidence of membrane permeabilization [8-14]. Recently, a new model, in which AmB forms an extramembraneous sponge layer that extracts ergosterol from cell membranes, was proposed [15]. The removal and sequestration of the ergosterol by AmB potentially interferes with all biological processes in the fungal cells that depend on ergosterol [16], and this was proposed to be the actual basis of the fungicidal activity of AmB. The new model is further supported by the fact that permeabilization does not necessarily lead to cell death [17] and that ergosterol-binding alone is sufficient to kill fungal cells [5]. However, the proposed ergosterol removal, and in particular its effect on the structure of the fungal cell membranes has not been directly investigated.

The structural effects of AmB on lipid membranes have been previously investigated using small angle neutron scattering [18] and neutron diffraction [19]. Both studies suggest that $\mathrm{AmB}$, when premixed with the very common membrane lipid 1-palmitoyl-2-oleoyl-sn-glycero3-phosphocholine (POPC), causes thicker membranes to form, and that this effect is larger when ergosterol is also present. AmB has also been shown to interact with POPC lipid monolayers at the air-water interface with different kinetics in the presence of ergosterol and cholesterol [20]. Such model lipid membranes made from one or two lipids are a first-line tool to investigate the function of cell membranes in more detail than is accessible in cell cultures. POPC is often used to represent the fluid lipid bilayer structure, and its relevance is motivated by the predominance of the phosphocholine headgroup and the two most abundant acyl chains, palmitoyl (C16:0) and oleyl (C18:1c9) in human and other mammalian cell membranes, as well as yeast. However, human and fungal cell membranes also contain a number of other phospholipid species and a broader distribution of acyl chains, all of which play a role in determining their physicochemical properties [21] and potential susceptibility to AmB. However, neutron scattering studies require the use of perdeuterated lipids (and/or sterols) for the detection of the sterols and AmB insertion. Per-deuterated POPC has not been available previously and was custom synthesized for this study in collaboration with the Australian National Deuteration Facility [22]. For the same reason, recent efforts have also been made toward obtaining neutron structures of deuterium-labeled yeast lipid membranes that more closely mimic the lipid composition of AmB's target cell membranes [23-26].

We recently investigated the interaction of $\mathrm{AmB}$ with mimics of fungal membranes consisting of supported lipid bilayers (SLBs) reconstituted from Pichia pastoris phospholipid extracts [27] using neutron reflectometry (NR) [28]. These membranes mimic the typical phospholipid composition of the fungal target membranes of AmB more closely than simple model membranes. Our results showed that AmB indeed forms an extramembraneous sponge layer as suggested [15] but also indicate that the layer is highly hydrated, consisting of 77-95\% water. We used per-deuterated yeast lipid extracts to determine that AmB inserts into these membranes in the absence and presence of ergosterol without forming observable aqueous pores. Our results were consistent with an AmB-induced extraction of deuterium labeled ergosterol into the AmB sponge layer and indicated that the membrane phospholipid composition had a significant effect on the consequence of the ergosterol removal. The presence of more polyunsaturated lipid fatty acid chains (particularly linoleic and linolenic) gave rise to a significant membrane thinning, which was not observed in membranes containing mainly mono-unsaturated lipid chains (oleic). Such a thinning could have a significant effect on membrane protein function and could also contribute to the efficiency of AmB in different types of membranes.

To consolidate our results on the yeast SLBs with results obtained by others using simple model membranes, we have now investigated the interaction of AmB with POPC-supported bilayers containing ergosterol or cholesterol under the same conditions. By multidimensional neutron contrast variation using deuterated $\mathrm{d}_{82}$ POPC [22] and deuterated ergosterol purified from yeast cell cultures, we show that although AmB inserts in both pure POPC as well as ergosterol- and cholesterol-containing POPC membranes, it only extracts ergosterol and not cholesterol. We also show that the AmB sponge layer observed on yeast extract membranes does not form on POPC-based model membranes. This indicates clearly that results obtained using model membranes depend on the lipid composition, 
and this needs to be further elucidated in order to understand lipid effects on the full mechanism of membrane-targeting amphiphilic drugs such as AmB.

\section{Materials and Methods}

All solvents and chemicals used in this work were analytical grade ( $\geq 99.0 \%)$ from Sigma Aldrich (Stockholm, Sweden/Sydney, Australia) and were used without further purification. 1-Palmitoyl-2-oleoyl-sn-glycero-3-phosphocholine (hPOPC) was purchased from Avanti Polar Lipids (Alabaster, AL, USA), and the per-deuterated $\mathrm{d}_{82}$ POPC ( $\mathrm{dPOPC}$ ) was synthesized as previously described [22] at the Australian National Deuteration Facility. Cholesterol (hChol) and ergosterol (hErg) were obtained from Sigma Aldrich (Stockholm, Sweden). Per-deuterated ergosterol $\mathrm{C}_{28} \mathrm{D}_{44} \mathrm{O}(\mathrm{dErg})$ was purified from per-deuterated lipid extracts of P. pastoris cultures grown at the Institut Laue Langevin D-Lab (Grenoble, France) as previously described [27], by precipitating twice from the apolar lipid fraction in the minimum amount of heptane. The purity of the dErg obtained $(\geq 99.0 \%)$ was determined by gas chromatography (GC-FID GC2010-Plus-Shimadzu, Stockholm, Sweden), and the degree of deuteration was confirmed by mass spectrometry (Agilent 6890 GC/5973N EI-MS—Agilent, Stockholm, Sweden) (Supplementary Materials Figure S1). Amphotericin $\mathrm{B} \mathrm{C}_{47} \mathrm{H}_{73} \mathrm{NO}_{17}$ (from Streptomyces sp., $\approx 80 \%$ ) was purchased from Sigma-Aldrich (Sydney, Australia) and used without further purification. Ultrapure $\mathrm{H}_{2} \mathrm{O}$ (MilliPore; resistivity $\left.>18 \mathrm{M} \Omega \cdot \mathrm{cm}\right)$ and $\mathrm{D}_{2} \mathrm{O}(99.8 \%$, Sigma Aldrich, Sydney, Australia) provided by the Australian Nuclear Science and Technology Organization (ANSTO) were used in all experiments.

Undoped $80 \times 50 \times 15 \mathrm{~mm}^{3}$ silicon single crystals polished on the (111) face to a typical roughness of $<3 \AA$ were purchased from Siltronix (Archamps, France). The silicon substrates were cleaned in an aqueous piranha solution $\left(5: 4: 1 \mathrm{H}_{2} \mathrm{O}: \mathrm{H}_{2} \mathrm{SO}_{4}: \mathrm{H}_{2} \mathrm{O}_{2}\right)$ for $15 \mathrm{~min}$ at $80^{\circ} \mathrm{C}$, followed by extensive rinsing with ultrapure water and UV/ozone cleaning (Jelight 144 AX-Jelight, Irvine, CA, USA) for $10 \mathrm{~min}$.

The lipid bilayers were formed by the vesicle fusion method, as previously described [29]. The lipids were dispersed in pure water using a tip sonicator (Sonopuls HD 3100-Bandelin, Berlin, Germany) by repeated pulses ( $3 \mathrm{~s}$ on, $5 \mathrm{~s}$ off) until the solutions became visibly clear ( $\approx 5 \mathrm{~min}$ ). Three $\mathrm{mL}$ of the vesicle solutions $(0.5 \mathrm{mg} / \mathrm{mL})$ were injected immediately after sonication into the neutron reflectivity sample cell equilibrated to $30^{\circ} \mathrm{C}$. This temperature was chosen in order to compare to our previous AmB results on yeast lipid membranes from cell cultures grown at $30^{\circ} \mathrm{C}$. The lipid solution was incubated for $30 \mathrm{~min}$ over the silicon substrates to allow the vesicles to fuse and spread on the crystal surface. Excess vesicles were removed by rinsing with $5 \mathrm{~mL} \mathrm{D}_{2} \mathrm{O}$, which was followed by exchange of the bulk solution contrast by rinsing with 15 to $20 \mathrm{~mL}$, corresponding to 10-15 times the volume of the sample cell, using an HPLC pump (Knauer 40P-Knauer Berlin, Germany). Four solvent contrasts (with their neutron scattering length densities (SLD) given in brackets) were used: $100 \% \mathrm{D}_{2} \mathrm{O}$ $\left(\mathrm{SLD} 6.35 \times 10^{-6} \AA^{-2}\right), \mathrm{CM} 4,\left(66 \% \mathrm{D}_{2} \mathrm{O}, \mathrm{SLD} 4 \times 10^{-6} \AA^{-2}\right), \mathrm{CMSi}\left(38 \% \mathrm{D}_{2} \mathrm{O}\right.$, contrast matched to the silicon substrates, SLD $\left.2.07 \times 10^{-6} \AA^{-2}\right)$, and $100 \% \mathrm{H}_{2} \mathrm{O}\left(\mathrm{SLD}-0.56 \times 10^{-6} \AA^{-2}\right)$.

$\mathrm{AmB}$ was first dissolved in DMSO at a concentration of $10 \mathrm{mM}$ and diluted by the addition of $\mathrm{D}_{2} \mathrm{O}$ to obtain a $1 \mathrm{mM}$ solution of 9:1 v/v, $\mathrm{D}_{2} \mathrm{O}$ :DMSO. This solution was sonicated for two minutes with the tip sonicator by repeated pulses ( $3 \mathrm{~s}$ on, 10s off) in order to obtain a clear yellow solution. A short sonication was performed before each sample injection. Once the lipid bilayer reflectivity had been measured in all solvent contrasts, $3 \mathrm{~mL}$ of the $1 \mathrm{mM} \mathrm{AmB}$ solution was injected over the bilayers. After 30 min of incubation, the bulk solution was rinsed off with 15 to $20 \mathrm{~mL}$ of pure $\mathrm{D}_{2} \mathrm{O}$ to remove the DMSO, which was followed by measurements in the same three to four solvent contrasts. Small differences in the neutron scattering length density value in each water contrast before and after AmB are a result of incomplete exchange of the bulk solution by the HPLC pump, unless otherwise stated.

Specular neutron reflection [30] measures reflectivity $\mathrm{R}$ as a function of the momentum transfer $\mathrm{q}=4 \pi \sin \theta / \lambda$, where $\theta$ is the angle of incidence and $\lambda$ is the wavelength of the neutron. The reflectivity is dependent on the structure of the interface and the coherent neutron scattering length density $\rho$ (SLD) of the materials. The SLD of a molecule is defined as the sum of the coherent scattering lengths 
of all atoms divided by the molecular volume. The thickness of the adsorbed layers and roughness of the interfaces also determine the reflectivity. By modeling the structure of the reflecting surface, in this case a supported membrane, as a series of layers corresponding to the molecular constituents, it is possible to determine the composition and one-dimensional structure of the SLB, as described previously [31]. By using deuterium labeling of the lipids and the solvent, NR allows the penetration of drugs and aqueous pore formation to be detected [32].

NR measurements were carried out on the Platypus neutron reflectometer at the Australian Centre for Neutron Scattering, ANSTO (Lucas Heights, Sydney, NSW, Australia) [33] using a neutron wavelength spectrum from 2.8 to $18 \AA$ at two incident angles $\left(0.8^{\circ}\right.$ and $\left.4^{\circ}\right)$ and constant wavelength resolution of $\Delta \lambda / \lambda=7 \%$. For measurements in $66 \%$ D2O contrasts, reflection was also measured at an additional angle $\left(0.6^{\circ}\right)$ in order to reach the low critical angle. This instrument setup allowed covering a q-range from 0.009 to $0.337 \AA^{-1}$ with reflectivity signals typically measurable above the incoherent sample background up to $0.25 \AA^{-1}$. The sample cell used for the measurements was a closed flow cell consisting of a $15 \mathrm{~mm}$ thick single crystal silicon wafer $(80 \mathrm{~mm} \times 50 \mathrm{~mm})$ mounted against a polyether ether ketone (PEEK) trough ( $1 \mathrm{~mL}$ internal volume) sandwiched between hollow aluminum holders to allow temperature control by means of water circulation. The cell was connected to a preparative HPLC pump (Knauer 40P) for mixing and injection of water contrasts.

Raw data were processed to subtract background and normalize data with transmission measurements [34]. Then, the NR data were analyzed by the simultaneous fitting of all solvent contrasts for each sample using the Motofit software package [34], which uses the Abeles optical matrix method [35]. To do this, a model corresponding to a series of homogeneous layers with thickness $\tau$, scattering length density $\rho$, and roughness $\sigma$ was constructed. The scattering length density of each layer is also influenced by solvent penetration, which was described by a volume fraction percentage, $\varphi$. These parameters were used to calculate the expected reflectivity of the model structures, and this was compared to the experimental data and refined until a good fit to all contrasts was obtained. Constraints were used for the selection of a physically realistic model, as described below.

The reflectivity of each silicon substrate was measured before the addition of lipids to determine the structure and roughness of the native silicon oxide layer $\mathrm{SiO}_{2} . \mathrm{All} \mathrm{SiO}_{2}$ layers $\left(\rho=3.41 \times 10^{-6} \AA^{-2}\right)$ of the different substrates were found to be of similar quality, with thicknesses of $10 \pm 4 \AA$, with $8 \pm 6 v / v \%$ solvent and a $3 \pm 2 \AA$ A roughness.

The phospholipid model structures were divided into layers corresponding to the polar head groups and the hydrophobic chains due to their different atomic constituents and solvent interaction. In order to define the molecular neutron scattering length densities (SLD) of these components, the carbonyl group was considered part of the head group volume and set as the interface between the two layers. Previously published molecular volumes of the lipid constituents as well as ergosterol, cholesterol, and AmB $[18,28,36-38]$ were used to calculate the SLD values shown in Table 1. The SLD of $\mathrm{AmB}$ increases with the deuterium content of the solvent because it contains 13 labile protons that exchange with the solvent.

The lipid bilayer structure was represented by a 3-layer model, the inner heads (facing the $\mathrm{SiO}_{2}$ ), the hydrophobic chains of both lipid leaflets, and the outer heads (facing the solution). The parameters of both head groups were not directly linked so as to allow different conformations toward the solution and silicon substrate interfaces, as this was found to improve the quality of the fits. The wet area per molecule $\left(\mathrm{A}_{\text {wet }}\right)$ of the lipid head groups and of the tails was calculated from the fitted layer thicknesses and solvent volume fractions and constrained to be the same for the heads and chains to within the fitting uncertainties given in the results tables. 
Table 1. Neutron scattering length densities and molecular volumes used in this study.

\begin{tabular}{ccccc}
\hline & POPC & Ergosterol & Cholesterol & AmB \\
\hline $\mathrm{V}\left(\AA^{3}\right)$ & Heads: $322^{\mathrm{a}}$ & $630^{\mathrm{c}}$ & $630^{\mathrm{c}}$ & $983^{\mathrm{c}}$ \\
& Chains: $934^{\mathrm{b}}$ & & & $2.88\left(\mathrm{D}_{2} \mathrm{O}\right)$ \\
$\rho\left(10^{-6} \AA^{-2}\right)^{\mathrm{d}}$ & Heads: $1.86(\mathrm{~h})$ & $0.43(\mathrm{~h})$ & $0.20(\mathrm{~h})$ & $2.41(\mathrm{CM} 4)$ \\
& $7.34(\mathrm{~d})$ & $7.70(\mathrm{~d})$ & & $2.01(\mathrm{CMSi})$ \\
$\rho\left(10^{-6} \AA^{-2}\right)$ & Chains: $-0.28(\mathrm{~h})$ & & & $1.50\left(\mathrm{H}_{2} \mathrm{O}\right)$ \\
\hline
\end{tabular}

a Volume of the glycerophosphocholine lipid headgroups, including the carbonyl groups and first carbon of the chains $\left(\mathrm{C}_{10} \mathrm{H}_{18} \mathrm{NO}_{8} \mathrm{P}\right)$ [36], b Volume of the lipid chains $\mathrm{C}_{32} \mathrm{H}_{64}$ [36], ${ }^{c}$ Molecular volume of ergosterol [18,28], cholesterol [38], and amphotericin B [18], ${ }^{\mathrm{d}}$ neutron scattering length density of the molecules calculated from the component/molecular volumes and isotopic composition $\left(\mathrm{h}=\right.$ nondeuterated moleucles, $\mathrm{d}=\mathrm{d}_{82}$-POPC calculated from NMR and mass spectroscopy [22] data, and perdeuterated $\mathrm{d}_{44}$-ergosterol as indicated by MS data-Supplementary Materials Figure S1).

In the sterol and AmB-containing bilayers, their volume fractions in the lipid chain region were taken into account in the area calculations. A water layer [39-41] between the $\mathrm{SiO}_{2}$ surface and the lipid bilayers was not required to obtain a good and physically realistic fit to the data. The volume fraction of solvent remaining in the lipid chains after the deposition of the bilayers ( $<6 \%$ in most cases) is related to a small number of defects in the bilayer over the large surface area $(\approx 65 \mathrm{~mm} \times 30 \mathrm{~mm})$ illuminated with neutrons and does not have an effect on the structural characterization of the bilayer. Quartz Crystal Microbalance measurements (Qsense E4 QCM-D Västra Frölunda, Sweden) showed that $\mathrm{AmB}$ did not interact with a clean $\mathrm{SiO}_{2}$ substrate surface (Supplementary Materials Figure S2).

The sterols were assumed to be oriented parallel to the phospholipid molecules, which is consistent with previously published data [31,42]. The exchangeable proton of the sterol hydroxyl group was not taken into consideration given the negligible effect on the SLD. A vertically homogeneous distribution of the sterols within the lipid chain region could be used to fit the data of all bilayers. The possibility of asymmetry in the SLD of the two lipid chain leaflets, corresponding to either lipid or sterol asymmetry [42] was investigated, but it was ruled out, as it did not improve the quality of the fits. Separation of the hydrophobic chain layer into several layers with different SLDs was also unfruitful, ruling out the proposed bilayer mid-plane location of cholesterol in polyunsaturated phosphocholine vesicles [43] and lower SLD layer at the bilayer center found in gel-phase 1,2-sn-dipalmitoyl phosphocholine (DPPC)-cholesterol bilayers [31].

After AmB addition, the final model structure was found using the corresponding fit to the bilayer structure before $\mathrm{AmB}$ as a starting point and allowing the head group and hydrophobic chain thicknesses and hydration to vary. The chain SLD of the lipids was also allowed to vary and could no longer be fitted to a constant value in the different solvent contrasts. However, the values were not a simple function of solvent penetration and could therefore only be due to the presence of $A m B$ exchangeable protons within the lipid chain region, which was used to determine AmB insertion into the bilayer. The amount of sterol removed by AmB could also be determined from simultaneous fitting of the solvent contrasts by requiring the amounts of sterol, lipid, AmB, and solvent to be constant.

The errors given for the fitted parameters in the results tables correspond to the maximum acceptable variation in each parameter that allowed a good fit to be maintained in the best solvent contrast for determining the given parameter, taking into account the physical constraints described above. The errors in the areas per molecule, sterol, and AmB mol fraction were propagated from the fitting uncertainties in the layer thickness, SLD, solvent fraction, sterol, and AmB volume fractions.

\section{Results}

In order to understand the changes due to $A m B$ interaction with the bilayers, NR data were recorded in the same solvent contrasts before and after interaction with the drug. hPOPC, its per-deuterated version $d_{82}$ POPC [22], ergosterol ( $\left.h E r g\right)$, per-deuterated $d_{44}$ ergosterol (dErg), and cholesterol (hChol) were used in order to distinguish between the sterols, the lipids, and the AmB. 
While the ergosterol-containing membranes represent a simple model of a fungal cell membrane, and the cholesterol-containing bilayers a mammalian membrane, it was also of interest to study the interaction of AmB with pure POPC bilayers to understand the effect of the sterols on the bilayer structure.

\subsection{AmB Interaction with Sterol-Free Bilayers: $h P O P C$ and $d_{82} P O P C$}

Figure 1 shows the reflectivity curves and scattering length density profiles corresponding to the best fits to the data for hPOPC and $d_{82}$ POPC bilayers measured in three contrasts $\left(\mathrm{D}_{2} \mathrm{O}, \mathrm{CM} 4\right.$ and $\mathrm{H}_{2} \mathrm{O}$ ) before and after interaction with AmB. The parameters of the fits and the associated fitting uncertainties are listed in Table 2. In addition to small differences in the substrate silicon dioxide layers, the data from both samples could be fitted with the same bilayer model.
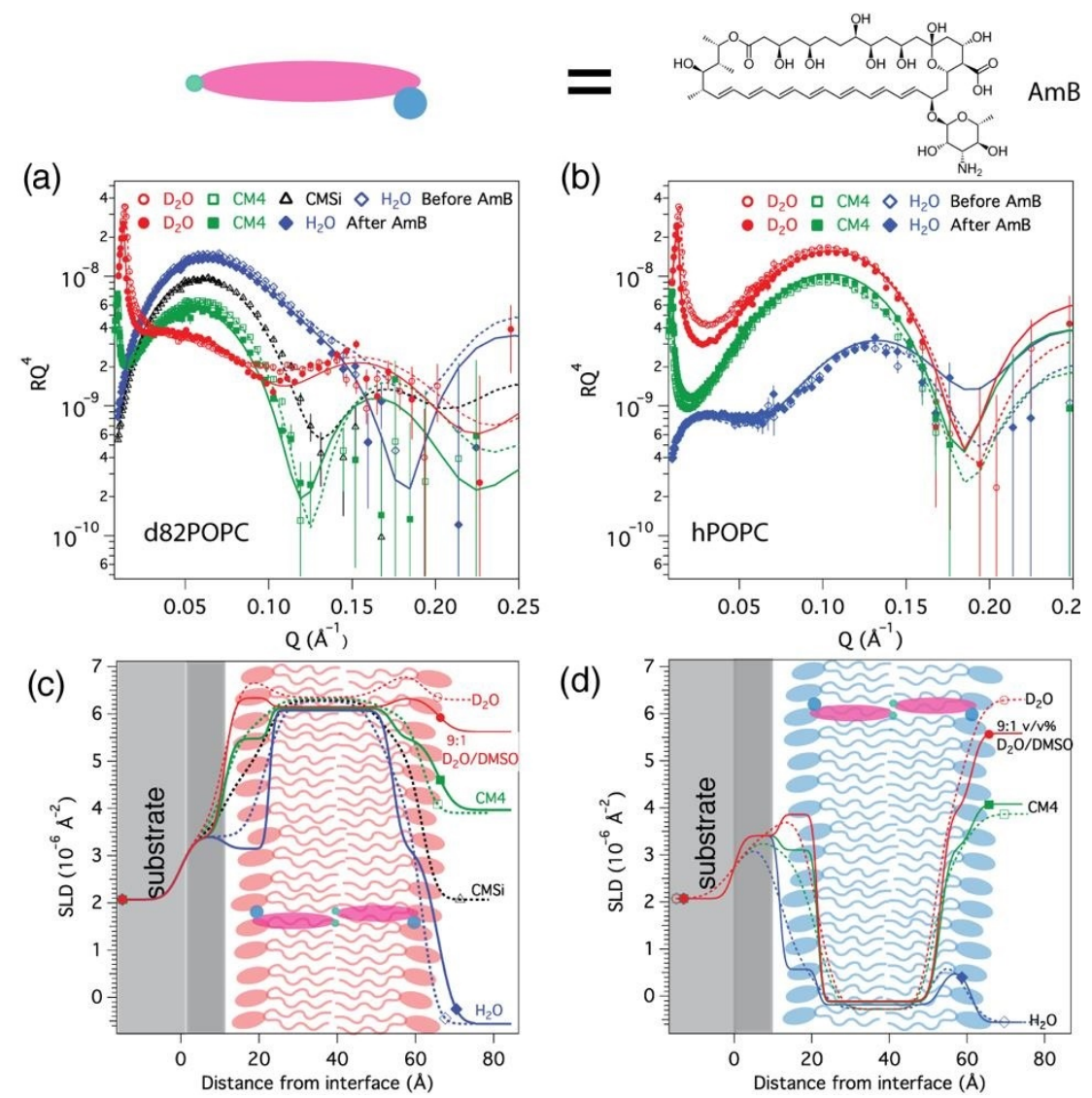

(d)

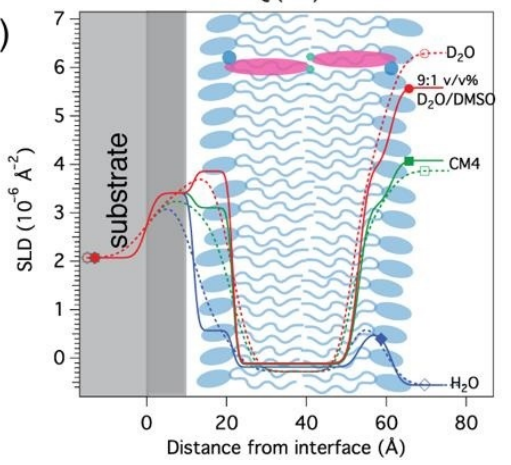

Figure 1. Neutron reflectivity data and the corresponding neutron scattering length density profiles of $\mathrm{d}_{82}$ POPC (1-palmitoyl-2-oleoyl-sn-glycero-3-phosphocholine) (a,c) and hPOPC (b,d) bilayers measured in different solvent contrasts at $30^{\circ} \mathrm{C}$ before (open symbols/dotted lines) and after (solid symbols/lines) addition of $1 \mathrm{mM}$ amphotericin $\mathrm{B}(\mathrm{AmB})$. A schematic illustration of the membrane structure and location of $\mathrm{AmB}$ corresponding to the fits is presented over the scattering length densities (SLD) profile (c,d). 
Table 2. Parameters corresponding to the best fit to the data from $\mathrm{d}_{82}$ POPC and hPOPC membranes before and after the addition of AmB addition, as displayed in Figure 1.

\begin{tabular}{|c|c|c|c|c|c|c|c|c|c|c|}
\hline \multicolumn{6}{|c|}{ Before AmB Addition } & \multicolumn{5}{|c|}{ After AmB Addition } \\
\hline & $\tau(\AA ̊)$ & $\rho\left(10^{-6} \AA^{-2}\right)$ & $\varphi(\%)$ & $\sigma(\AA)$ & $A\left(\AA^{2}\right)$ & $\tau(\AA ̊)$ & $\rho\left(10^{-6} \AA^{-2}\right)$ & $\varphi(\%)$ & $\sigma(\AA)$ & $A\left(\AA^{2}\right)$ \\
\hline$d_{82} P$ & & & & & & \multicolumn{5}{|c|}{$5.5 \pm 0.9 \mathrm{~mol} \% \mathrm{AmB}$ (heads and chains) } \\
\hline Inner heads & $11 \pm 1$ & $7.35 \pm 0.2$ & $0 \pm 5$ & $4 \pm 1$ & 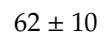 & $11 \pm 1$ & $7.08 \pm 0.1^{\mathrm{a}}$ & $51 \pm 5$ & $2 \pm 1$ & $63 \pm 12$ \\
\hline Chains & $31 \pm 1$ & $6.35 \pm 0.05$ & $1 \pm$ & $4 \pm$ & 61 & $32 \pm 1$ & $6.15 \pm 0.04^{b}$ & $0 \pm 1$ & $1 \pm 1$ & $63 \pm 3$ \\
\hline Outer heads & $9 \pm 1$ & $7.35 \pm 0.2$ & $43 \pm 5$ & $4 \pm 1$ & $63 \pm 10$ & $11 \pm 1$ & $7.08 \pm 0.1^{\mathrm{a}}$ & $51 \pm 5$ & $2 \pm 1$ & $63 \pm 12$ \\
\hline hPOPC & & & & & & \multicolumn{5}{|c|}{$5.5 \pm 0.9 \mathrm{~mol} \%$ AmB (chains) } \\
\hline Inner heads & $10 \pm 1$ & $1.86 \pm$ & $46 \pm 5$ & $4 \pm$ & 63 & $10 \pm 1$ & $1.86 \pm 0.2^{\mathrm{d}}$ & $53 \pm 5$ & $3 \pm 1$ & $74 \pm 15$ \\
\hline Chail & $30 \pm 1$ & $-0.28 \pm$ & $0 \pm$ & & & $32 \pm 1$ & $-0.106 \pm 0.06^{c}$ & $0 \pm 1$ & $1 \pm 1$ & $63 \pm 3$ \\
\hline Outer heads & $10 \pm 1$ & $1.86 \pm 0.2$ & $46 \pm 5$ & $3 \pm 1$ & $63 \pm 15$ & $8 \pm 1$ & $1.86 \pm 0.2^{\mathrm{d}}$ & $57 \pm 5$ & $3 \pm 1$ & $99 \pm 15$ \\
\hline
\end{tabular}

$\tau=$ layer thickness; $\rho=$ coherent neutron scattering length density of the layers without the solvent contribution; $\varphi=$ solvent volume fraction; $\sigma=\sigma$-value of a Gaussian interfacial roughness between each layer and the previous layer; $\mathrm{A}$ = wet area per lipid molecule and the associated water calculated from the molecular component volumes shown in Table 1. The volume occupied by AmB was added to the lipid volume for the lipid area (A) calculation. The $\rho$ value of the lipid chains and headgroup varied with solvent contrast after $A m B$ addition. ${ }^{a}$ In $D_{2} \mathrm{O}$, $7.05 \pm 0.08 \times 10^{-6} \AA^{-2}$ in CM4 and $7.01 \pm 0.06 \times 10^{-6} \AA^{-2}$ in $\mathrm{H}_{2} \mathrm{O} ;{ }^{\mathrm{b}}$ In $\mathrm{D}_{2} \mathrm{O}, 6.13 \pm 0.07 \times 10^{-6} \AA^{-2}$ in CM4 and $6.07 \pm 0.08 \times 10^{-6} \AA^{-2}$ in $\mathrm{H}_{2} \mathrm{O},{ }^{c}$ in $\mathrm{D}_{2} \mathrm{O},-0.128 \pm 0.06 \times 10^{-6} \AA^{-2}$ in $\mathrm{CM} 4$ and $-0.186 \pm 0.05 \times 10^{-6} \AA^{-2}$ in $\mathrm{H}_{2} \mathrm{O}$. ${ }^{\mathrm{d}}$ The variation in the hydrogenous headgroup SLD due to AmB was within the fitting uncertainties and not included in the fit.

The structure of the POPC $/ \mathrm{d}_{82}$ POPC bilayers correlates well with previously published structures $[44,45]$ in terms of headgroup $(10 \pm 1 \AA)$ and chain region thicknesses $(31 \pm 1 \AA)$, with no observable water in the chain region. The best fit to the data before $\mathrm{AmB}$ addition resulted in a dry area per lipid molecule of $62 \pm 3 \AA^{2}$, which is in good agreement with the previously published value of $64.3 \pm 1.3 \AA^{2}$, as determined by small angle scattering for POPC vesicles at $30^{\circ} \mathrm{C}$ [46].

The changes induced by AmB in the pure POPC bilayers were rather modest. The change of critical angle and the SLD of $\mathrm{D}_{2} \mathrm{O}$ observed on $\mathrm{AmB}$ injection in $\mathrm{D}_{2} \mathrm{O}$ arises from the presence of DMSO in the AmB solution injected, which, as also previously reported by Dabkowska et al. [47], was found to have no effect on the bilayer structure and was rinsed away before subsequent measurements.

The main consequence of AmB addition was the modification of the hydrophobic chain layer SLD. The lipid chain SLD was allowed to vary with solvent contrast, but the result could not be explained by only solvent penetration into the chains. Thus, the SLD of the chain layer was modeled considering the 13 exchangeable protons of $A m B$, which means that the SLD of the chains should change from $\mathrm{H}_{2} \mathrm{O}$ to $\mathrm{D}_{2} \mathrm{O}$ contrasts in a way that corresponds to the amount of $\mathrm{AmB}$ in that region. As AmB has SLD values in the range of $1.5-2.9 \times 10^{-6} \AA^{-2}$ depending on the solvent (Table 1), the SLD of hPOPC chains in all solvent contrasts should increase, while the SLD of deuterated $d_{82}$ POPC should decrease with AmB insertion. It was also necessary to allow variation of the deuterated lipid headgroup SLD with solvent contrast and adjust the lipid headgroup area by the corresponding volume of AmB. For the hydrogenous POPC headgroup, the SLD changes were within the fitting uncertainties due to the small difference in $\mathrm{AmB}$ and glycerophosphocholine SLD values (Table 1).

For $\mathrm{d}_{82}$ POPC, the SLD of the chain layer decreased after AmB addition from $6.35 \pm 0.0510^{-6} \AA^{-2}$ to $6.07 \pm 0.0410^{-6} \AA^{-2}$ in the most sensitive contrast, $\mathrm{H}_{2} \mathrm{O}$. This corresponds to $5.8 \pm 1.0 \% v / v$ $(5.5 \pm 0.9 \mathrm{~mol} \%)$ of AmB relative to the lipids. For hPOPC, the contrast of the lipid chains to the hydrogenous AmB molecules is lower than in the per-deuterated membrane, but a small increase in SLD could still be observed from $-0.28 \pm 0.0510^{-6} \AA^{-2}$ to $-0.106 \pm 0.0610^{-6} \AA^{-2}$ in the $\mathrm{D}_{2} \mathrm{O}$ contrast, which is consistent with the $5.8 \pm 2.0 \% v / v(5.5 \pm 1.9 \mathrm{~mol} \%)$ of $\mathrm{AmB}$ found in the deuterated bilayer. The area per molecule for the lipid chains did not change significantly from the pure lipid bilayers, as the amount of AmB inserted was small. However, the interfacial roughness values decreased, and there was a small increase in the lipid chain thickness. This indicates that AmB insertion has a small ordering effect on the lipids. An increase in the headgroup hydration of hydrogenous POPC previously reported after $\mathrm{AmB}$ addition to monolayers $(+28 \pm 3 v / v \%)$ by Foglia et al. [20] was also observed here; however, the data from the deuterated $\mathrm{d}_{82}$ POPC shows that a part of $\mathrm{AmB}$ is also present in the 
headgroup region, as the SLD of the deuterated phoshocholine headgroups was found to be lower than in the absence of AmB (Table 2). Therefore, the apparently higher headgroup hydration/area in $\mathrm{hPOPC}$ appears to originate from the poor contrast to AmB molecules, which does not allow assigning the change to the headgroup SLD outside the fitting uncertainties $\left( \pm 0.2 \times 10^{-6} \AA^{-2}\right)$. Finally, no water insertion that could have indicated aqueous pore formation $[8,9]$ could be observed in either hPOPC or $\mathrm{d}_{82}$ POPC.

The amount of AmB inserted into POPC bilayers is of the same order of magnitude as that observed previously in reconstituted yeast phospholipid bilayers [28], but the polyunsaturated yeast SLBs became thinner after AmB insertion in contrast to POPC, which appears to become slightly thicker. As the yeast phospholipid mixtures also contain phosphatidyl-ethanolamine (PE), phosphatidylserine (PS), phosphatidylinositol (PI), cardiolipin (CL), and phosphatidylglycerol (PG) in addition to phosphatidyl choline (PC) with a range of chain lengths and unsaturations, it is clear that the lipid composition has an effect on the AmB-bilayer interaction. In these POPC bilayers, we could also not observe an additional layer of AmB on top of the membranes, which was always the case with yeast phospholipids. This implies that the sponge layer formation is not a prerequisite for AmB insertion into POPC bilayers.

\subsection{AmB Interaction with Ergosterol-Containing Bilayers}

Bilayers containing nominally $15 \mathrm{~mol} \%$ ergosterol relative to the lipids were formed, similar to previously investigated $P$. pastoris phospholipid membranes. The actual ergosterol content in the supported bilayers was determined from the observed lipid chain SLD values, and it varies somewhat from the nominal value due to experimental uncertainties and the precision relating to data analysis uncertainties. We used several different membrane contrasts, which contained either ergosterol (hErg) or per-deuterated $\mathrm{d}_{44}$ ergosterol ( $\mathrm{dErg}$ ), with the aim to determine how the ergosterol initially present in the bilayer changes in response to $\mathrm{AmB}$ and how the lipid bilayer structure is modified as a consequence. Due to the isotopic composition of the POPC-ergosterol samples, each membrane and solvent contrast is sensitive to a different part of the system. To solve these structures, the data were fitted to the same global model as far as possible, respecting that small variations could arise from the preparation of different samples. The volume occupied by ergosterol, as determined from the fitted chain SLD values, was added to the lipid chain volume for the lipid area (A) calculation.

Figure 2 shows the reflectivity and SLD profiles corresponding to the best fits for hPOPC-dErg $(10 \pm 1 \mathrm{~mol} \%)$ and hPOPC-hErg $(14 \pm 6 \mathrm{~mol} \%)$ bilayers in three water contrasts $\left(\mathrm{D}_{2} \mathrm{O}, \mathrm{CM} 4\right.$ and $\left.\mathrm{H}_{2} \mathrm{O}\right)$, as well as $\mathrm{d}_{82}$ POPC-hErg $(19 \pm 2 \mathrm{~mol} \%)$, with the structural parameters given in Table 3 . The data for all three bilayers could be fitted with the same structural model even if the $\mathrm{d}_{82} \mathrm{POPC}-\mathrm{hErg}$ bilayer had a somewhat lower surface coverage $(95 \pm 3 \%)$. The lipid chain region had a very similar thickness of $32 \pm 1 \AA$ as in pure POPC bilayers, indicating that these low quantities of ergosterol do not have a significant thickening effect. The bilayers contained a small amount of solvent (2-6 v/v\%) indicating the presence of some defects, but the corresponding dry area per lipid $\left(62 \pm 1 \AA^{2}\right)$ was the same as in the ergosterol-free bilayers, indicating no significant ordering effect of the ergosterol. This is in good agreement with recently published data on the moderate condensing effect of ergosterol in POPC bilayers [48] that is quite different from the much stronger effect of cholesterol. At $30 \mathrm{~mol} \%$ ergosterol, a larger increase in the thickness $(4 \pm 1 \AA)$ of the chain region of POPC and a reduction in the hydration of the head groups $(10 \pm 3 \%)$ have been reported in monolayers [20] and in multilamellar vesicles [18], but we did not observe such a major difference in the $\mathrm{d}_{82}$ POPC-hErg sample containing $19 \pm 2 \mathrm{~mol} \%$ ergosterol. 


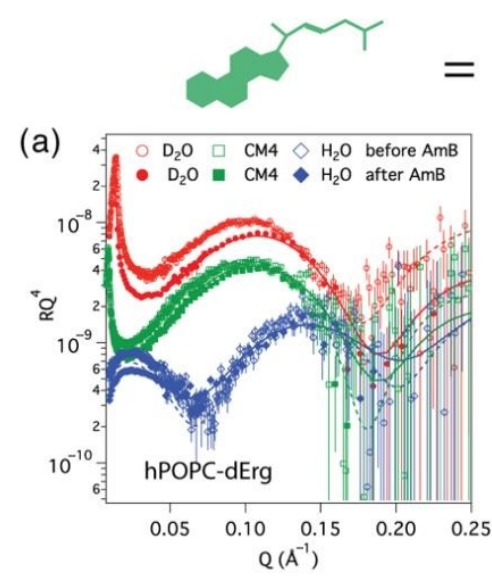

$=\operatorname{hERg}=\mathrm{dERg}$
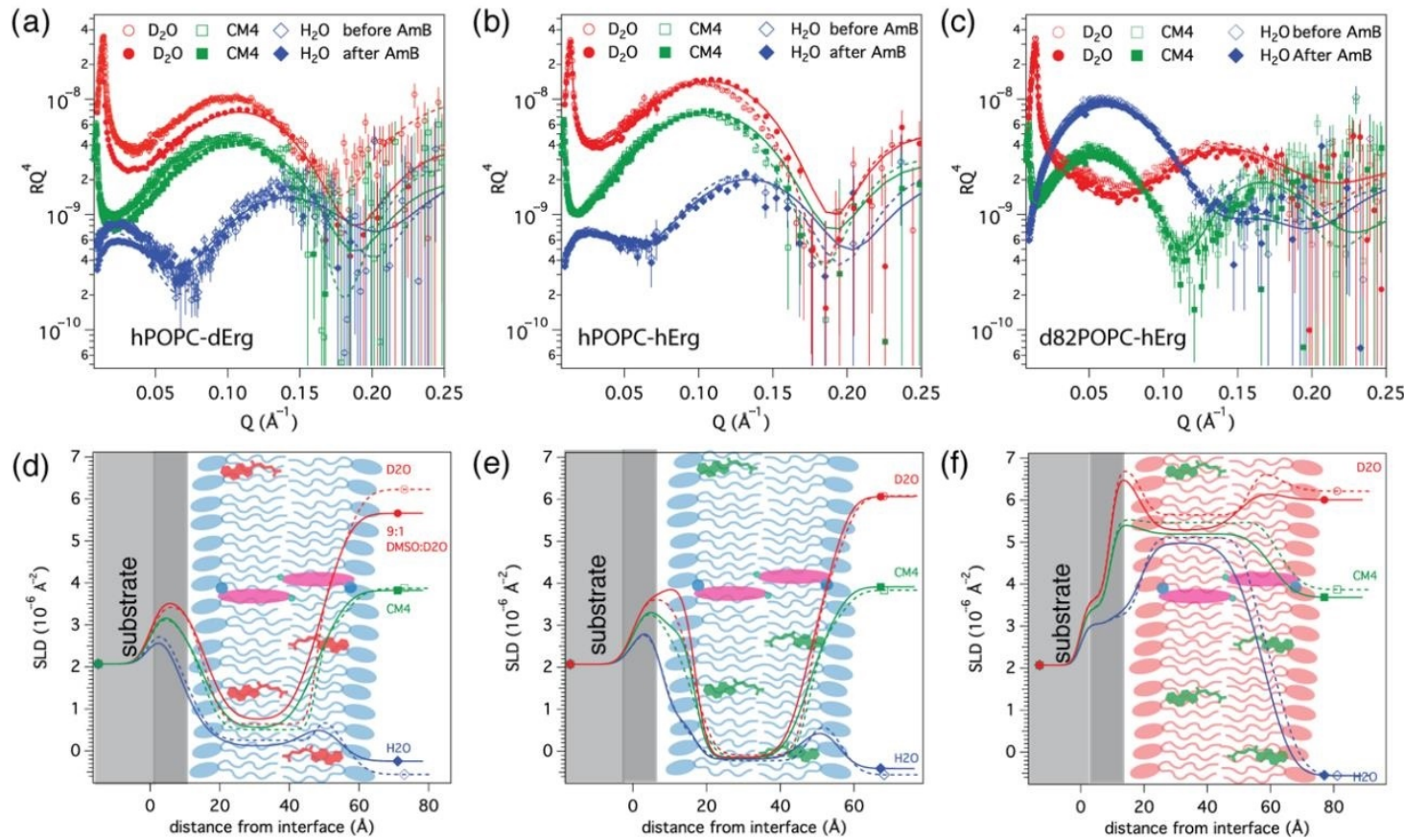

Figure 2. Neutron reflectivity data and the corresponding neutron scattering length density profiles of hPOPC-dErg (a,d) and hPOPC-hErg (b,e) and $\mathrm{d}_{82}$ POPC-hErg (c,f) bilayers measured in different solvent contrasts at $30^{\circ} \mathrm{C}$ before (open symbols/dotted lines) and after (solid symbols/lines) addition of $1 \mathrm{mM}$ AmB. A schematic illustration of the membrane structure and location of ergosterol and AmB corresponding to the fits is presented over the SLD profile $(\mathbf{d}-\mathbf{f})$. dErg: per-deuterated $\mathrm{d}_{44}$ ergosterol, hErg: ergosterol.

Table 3. Parameters corresponding to the best fits to the data from POPC-ergosterol bilayers before and after AmB addition, as displayed in Figure 2.

\begin{tabular}{|c|c|c|c|c|c|c|c|c|c|c|}
\hline \multicolumn{6}{|c|}{ Before AmB Addition } & \multicolumn{5}{|c|}{ After AmB Addition } \\
\hline & $\tau(\AA ̊)$ & $\rho\left(10^{-6} \AA^{-2}\right)$ & $\varphi(\%)$ & $\sigma(\AA)$ & $A\left(\AA^{2}\right)$ & $\tau(\AA)$ & $\rho\left(10^{-6} \AA^{-2}\right)$ & $\varphi(\%)$ & $\sigma(\AA)$ & $A\left(\AA^{2}\right)$ \\
\hline hPOPC-dErg & \multicolumn{5}{|c|}{$10 \pm 1 \mathrm{~mol} \% \mathrm{dErg}$} & \multicolumn{5}{|c|}{$3.0 \pm 2.8 \mathrm{~mol} \% \mathrm{AmB} \& 7.4 \pm 1.5 \mathrm{~mol} \% \mathrm{Erg}$} \\
\hline Inner heads & $8 \pm 1$ & $1.86 \pm 0.20$ & $36 \pm 8$ & $3 \pm 2$ & $67 \pm 13$ & $8 \pm 1$ & $1.86 \pm 0.20$ & $47 \pm 7$ & $3 \pm 2$ & $76 \pm 12$ \\
\hline Chains & $32 \pm 1$ & $0.3 \pm 0.07$ & $6 \pm 2$ & $3 \pm 2$ & $67 \pm 4$ & $30 \pm 1$ & $0.20 \pm 0.06^{\mathrm{a}}$ & $10 \pm 2$ & $5 \pm 2$ & $76 \pm 5$ \\
\hline Outer heads & $8 \pm 1$ & $1.86 \pm 0.20$ & $36 \pm 8$ & $4 \pm 2$ & $67 \pm 13$ & $8 \pm 1$ & $1.86 \pm 0.20$ & $44 \pm 8$ & $5 \pm 2$ & $76 \pm 12$ \\
\hline hPOPC-hErg & \multicolumn{5}{|c|}{$14 \pm 6 \mathrm{~mol} \% \mathrm{hErg}$} & \multicolumn{5}{|c|}{$4.2 \pm 3.8 \mathrm{~mol} \% \mathrm{AmB} \& 7.7 \pm 5.8 \mathrm{~mol} \% \mathrm{Erg}$} \\
\hline Inner heads & $9 \pm 1$ & $1.86 \pm 0.20$ & $42 \pm 9$ & $3 \pm 2$ & $65 \pm 12$ & $9 \pm 1$ & $1.86 \pm 0.20$ & $49 \pm 8$ & $3 \pm 2$ & $70 \pm 12$ \\
\hline Chains & $32 \pm 1$ & $-0.22 \pm 0.04$ & $2 \pm 2$ & $4 \pm 2$ & $64 \pm 4$ & $30 \pm 1$ & $-0.14 \pm 0.06^{\mathrm{b}}$ & $2 \pm 2$ & $3 \pm 2$ & $68 \pm 5$ \\
\hline Outer heads & $9 \pm 1$ & $1.86 \pm 0.20$ & $42 \pm 9$ & $3 \pm 2$ & $65 \pm 12$ & $9 \pm 1$ & $1.86 \pm 0.20$ & $48 \pm 8$ & $5 \pm 2$ & $72 \pm 12$ \\
\hline $\mathrm{d}_{82}$ POPC-hErg & \multicolumn{5}{|c|}{$19 \pm 2 \mathrm{~mol} \% \mathrm{hErg}$} & \multicolumn{5}{|c|}{$21.7 \pm 6.8 \mathrm{~mol} \%$ AmB \& $8.4 \pm 4.7 \mathrm{~mol} \% \mathrm{Erg}$} \\
\hline Inner heads & $10 \pm 1$ & $7.35 \pm 0.20$ & $52 \pm 8$ & $2 \pm 2$ & $67 \pm 11$ & $10 \pm 1$ & $7.35 \pm 0.20$ & $52 \pm 8$ & $2 \pm 2$ & $75 \pm 13$ \\
\hline Chains & $34 \pm 1$ & $5.60 \pm 0.07$ & $8 \pm 2$ & $3 \pm 2$ & $67 \pm 4$ & $32 \pm 1$ & $5.17 \pm 0.09^{\mathrm{c}}$ & $0 \pm 2$ & $4 \pm 2$ & $75 \pm 3$ \\
\hline Outer heads & $9 \pm 1$ & $7.35 \pm 0.20$ & $47 \pm 8$ & $3 \pm 2$ & $67 \pm 11$ & $9 \pm 1$ & $6.81^{\mathrm{d}} \pm 0.20$ & $49 \pm 9$ & $6 \pm 2$ & $75 \pm 16$ \\
\hline
\end{tabular}

The effect of AmB on the structure of POPC-ergosterol bilayers was more pronounced than for pure POPC bilayers, although the changes in neutron reflectivity were much smaller than in yeast lipid bilayers [28]. In the hPOPC-dErg sample, AmB caused the lipid chain SLD to decrease in all contrasts, showing clearly that some of the deuterated ergosterol was extracted from the bilayer. The chain SLD was also dependent on the solvent contrast, and it corresponded to $3.2 \pm 2.9 v / v \%(3.0 \pm 2.8 \mathrm{~mol} \%)$ $\mathrm{AmB}$ in the lipid chains, which was somewhat lower than that in pure POPC bilayers. The volume 
of ergosterol remaining $(5.0 \pm 1.0 \mathrm{v} / \mathrm{v} \%$ or $7.4 \pm 1.5 \mathrm{~mol} \% \mathrm{Erg})$ corresponds to $73 \%$ of the ergosterol originally present in the membrane. The volume of AmB inserted is similar to the volume of ergosterol removed. The remaining amount of ergosterol and that of AmB inserted are approximately in a 2:1 ratio, which is opposed to the previously proposed [49] formation of an approximately 1:1 complexes. Considering the fitting uncertainties, the solvent content in this sample very slightly increased from $6 \pm 2 v / v \%$ to $10 \pm 3 v / v \%$ by AmB addition, which correlates roughly with the volume of ergosterol extracted, but this may not necessarily represent pore formation, as the same was not observed in any of the other samples.

In hPOPC-hErg, although the neutron contrast to ergosterol removal from the lipid chains is much lower, the AmB effect was consistent with the results obtained with dErg, with $57 \%$ of the ergosterol remaining $(5.2 \pm 3.9 \% v / v)$ and $A m B$ insertion of $4.4 \pm 4.0 v / v \%$ relative to the lipids, without water insertion.

In the $\mathrm{d}_{82}$ POPC-hErg membrane $(19 \pm 2 \mathrm{~mol} \%)$, the fraction of ergosterol remaining was $45 \%$ of the original, and the amount extracted was higher, as was the amount of AmB inserted $(21.7 \pm 6.8 \mathrm{~mol} \%)$. However, this bilayer also had a lower initial surface coverage $(92 \pm 2 \%)$, and the insertion of $\mathrm{AmB}$ also removed the water fraction, which suggests that some of the pre-existing defects were filled by $A m B$ or the bilayer expanded during the insertion. We note that AmB on its own has no irreversible interaction with the clean silicon substrate, as determined with QCM-D measurements (Supporting Information Figure S2). Only the outer headgroup region SLD of $\mathrm{d}_{82}$ POPC-hErg decreased after AmB addition, which would correspond to $12 v / v \%$ AmB, but part of this change could also be due to the presence of hErg during the extraction and thus can not be uniquely assigned.

Thus, the main effect of AmB was the removal of a significant proportion of the ergosterol and insertion into the lipid chain region. AmB insertion seemed to depend to some degree on the initial lipid surface coverage.

\subsection{AmB Interaction with Cholesterol-Containing Bilayers}

The data from the two cholesterol-containing samples, $\mathrm{d}_{82}$ POPC-hChol and hPOPC-hChol are presented in Figure 3. Both could be fitted with a similar model of the bilayer structure (Table 4 ) with a scattering length density profile corresponding closely to the nominal cholesterol content $(18 \pm 3 \mathrm{~mol} \%$ for $\mathrm{d}_{82}$ POPC-hChol and $15 \pm 8 \mathrm{~mol} \%$ for hPOPC-hChol). The hydrophobic chain region thickness (33 $\pm 1 \AA$ ) was somewhat higher than for the pure POPC and POPC-ergosterol bilayers and consistent with the $2 \AA$ thickening caused by $15 \mathrm{~mol} \%$ cholesterol in POPC bilayers previously observed by lamellar X-ray diffraction [48]. Table 4 lists the wet areas per molecule that include the area occupied by the cholesterol and solvent fractions. Although both bilayers contained a small amount of solvent-filled defects, the dry area calculated from this per POPC molecule without the cholesterol $\left(55 \pm 4 \AA^{2}\right)$ is consistent with previous reports showing that cholesterol has a higher ordering effect than ergosterol in POPC bilayers [50]. The cholesterol was assumed to be oriented parallel to the phospholipids [19], as only homogeneous models of the hydrophobic region SLD fit the data satisfactorily. We could not fit our data with an asymmetry in the SLD of the lipid chain leaflets in a similar manner to recently reported in deuterated PC from E. coli [42], which contains a range of different chains.

In contrast to the ergosterol-containing bilayers, in the $\mathrm{d}_{82} \mathrm{POPC}-\mathrm{hChol}$ sample, it could be clearly seen that much more AmB $(11.7 \pm 3.5 v / v \%)$ inserted in the bilayer, and no cholesterol was removed within the detection limit. The SLD of both headgroup regions decreased, as observed also for pure $\mathrm{d}_{82}$ POPC. The AmB insertion was accompanied by a reduction in the water content of the lipid chains and also resulted in a small thickening $(\approx 1 \AA)$ of the acyl chains and reduced interfacial roughness. The hPOPC-hChol lipid bilayer contrast to cholesterol removal is lower, but a small change was observed in the lipid chain SLD, which is consistent with $5.4 \pm 2.5 v / v \%$ AmB insertion and no cholesterol removal. These results are consistent with the notion that at these relatively low 
amounts of sterols at least, AmB does not extract any cholesterol, and the amount of AmB inserted is correspondingly higher than in pure POPC or ergosterol-containing bilayers.
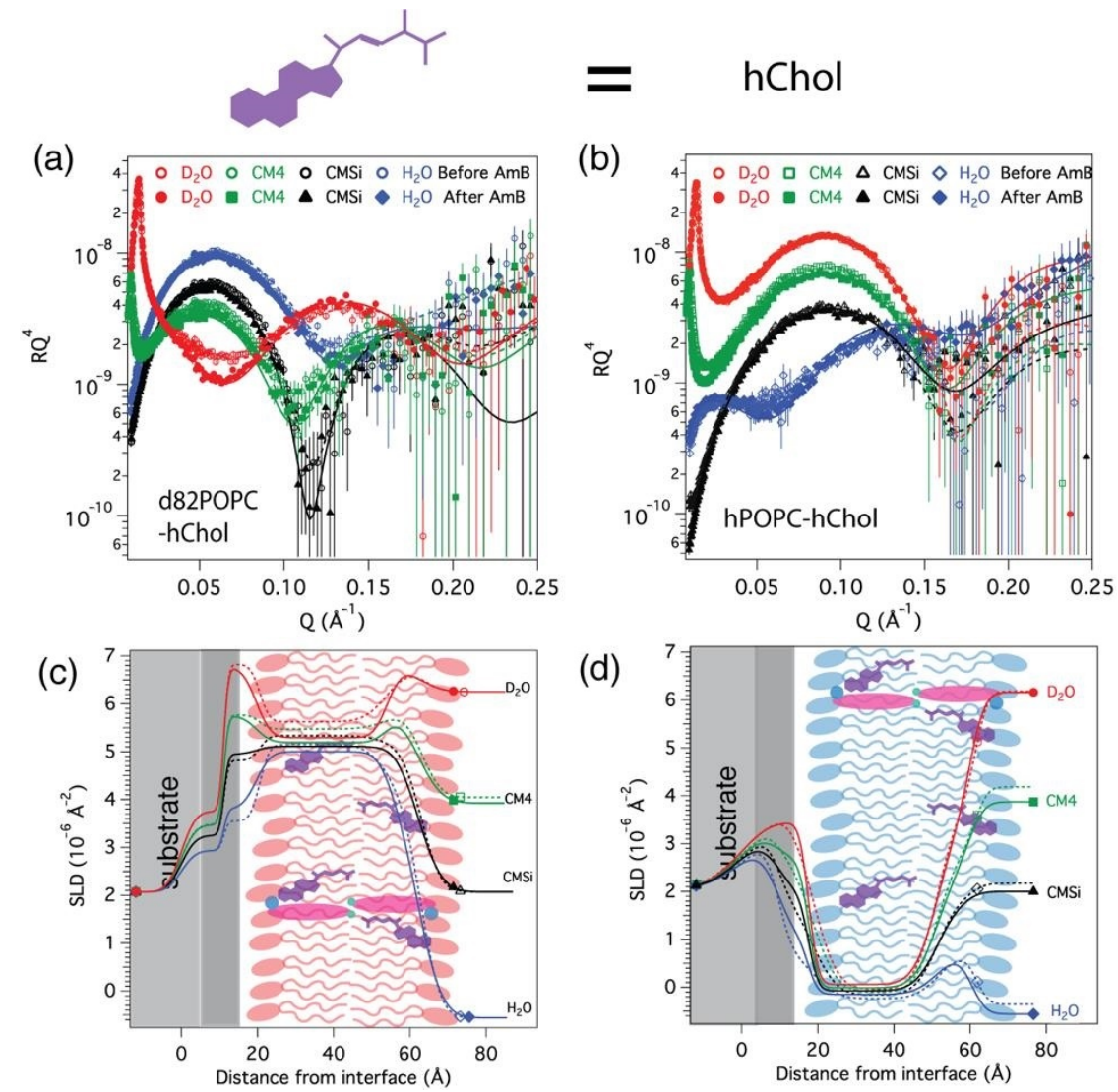

Figure 3. Neutron reflectivity data and the corresponding neutron scattering length density profiles of $\mathrm{d}_{82}$ POPC-hChol $(\mathbf{a}, \mathbf{c})$ and $\mathrm{hPOPC}-\mathrm{hChol}(\mathbf{b}, \mathbf{d})$ bilayers measured in different solvent contrasts at $30{ }^{\circ} \mathrm{C}$ before (open symbols/dotted lines) and after (solid symbols/lines) addition of $1 \mathrm{mM}$ of AmB. A schematic illustration of the membrane structure and location of cholesterol and AmB corresponding to the fits is presented over the SLD profile $(\mathbf{c}, \mathbf{d})$.

Table 4. Parameters corresponding to the best fits to the data from POPC-cholesterol bilayers before and after AmB addition, as displayed in Figure 3.

\begin{tabular}{|c|c|c|c|c|c|c|c|c|c|c|}
\hline \multicolumn{6}{|c|}{ Before AmB Addition } & \multicolumn{5}{|c|}{ After AmB Addition } \\
\hline & $\tau(\AA ̊)$ & $\rho\left(10^{-6} \AA^{-2}\right)$ & $\varphi(\%)$ & $\sigma(\AA)$ & $A\left(\AA^{2}\right)$ & $\tau(\AA)$ & $\rho\left(10^{-6} \AA^{-2}\right)$ & $\varphi(\%)$ & $\sigma(\AA)$ & $A\left(\AA^{2}\right)$ \\
\hline $\mathrm{d}_{82}$ POPC-hChol & \multicolumn{5}{|c|}{$18.3 \pm 3 \mathrm{~mol} \%$ hChol } & \multicolumn{5}{|c|}{$11.1 \pm 3.3 \mathrm{~mol} \%$ AmB \& $18.4 \pm 3.4 \mathrm{~mol} \% \mathrm{hChol}$} \\
\hline Inner heads & $10 \pm 1$ & $7.35 \pm 0.20$ & $48 \pm 8$ & $1 \pm 2$ & $66 \pm 9$ & $8 \pm 1$ & $7.15 \pm 0.20^{\mathrm{a}}$ & $43 \pm 8$ & $1 \pm 2$ & $68 \pm 11$ \\
\hline Chains & $34 \pm 1$ & $5.58 \pm 0.05$ & $7 \pm 3$ & $2 \pm 2$ & $66 \pm 4$ & $35 \pm 1$ & $5.27 \pm 0.20^{b}$ & $2 \pm 2$ & $2 \pm 2$ & $68 \pm 3$ \\
\hline Outer heads & $9 \pm 1$ & $7.35 \pm 0.20$ & $44 \pm 8$ & $5 \pm 2$ & $66 \pm 9$ & $9 \pm 1$ & $7.15 \pm 0.20^{\mathrm{a}}$ & $37 \pm 9$ & $3 \pm 2$ & $68 \pm 12$ \\
\hline hPOPC-hChol & \multicolumn{5}{|c|}{$14.5 \pm 8 \mathrm{~mol} \%$ hChol } & \multicolumn{5}{|c|}{$5.7 \pm 2.6 \mathrm{~mol} \% \mathrm{AmB} \& 14.5 \pm 3.7 \mathrm{~mol} \% \mathrm{hChol}$} \\
\hline Inner heads & $9 \pm 1$ & $1.86 \pm 0.20$ & $45 \pm 7$ & $3 \pm 2$ & $65 \pm 7$ & $8 \pm 1$ & $1.8 \pm 0.20$ & $38 \pm 7$ & $4 \pm 2$ & $65 \pm 10$ \\
\hline Chains & $33 \pm 1$ & $-0.23 \pm 0.03$ & $3 \pm 2$ & $5 \pm 2$ & $65 \pm 3$ & $34 \pm 1$ & $-0.07 \pm 0.06^{c}$ & $2 \pm 2$ & $2 \pm 2$ & $65 \pm 4$ \\
\hline Outer heads & $10 \pm 1$ & $1.8 \pm 0.20$ & $51 \pm 7$ & $5 \pm 2$ & $65 \pm 7$ & $9 \pm 1$ & $1.8 \pm 0.20$ & $45 \pm 7$ & $5 \pm 2$ & $69 \pm 10$ \\
\hline
\end{tabular}

${ }^{\mathrm{a}}$ In $\mathrm{D}_{2} \mathrm{O}, 7.12 \pm 0.05 \times 10^{-6} \AA^{-2}$ in CM4, $7.11 \pm 0.06 \times 10^{-6} \AA^{-2}$ in CMSi and $7.09 \pm 0.08 \times 10^{-6} \AA^{-2}$ in $\mathrm{H}_{2} \mathrm{O}^{\mathrm{b}}$ In $\mathrm{D}_{2} \mathrm{O}$, $5.22 \pm 0.05 \times 10^{-6} \AA^{-2}$ in CM4, $5.17 \pm 0.06 \times 10^{-6} \AA^{-2}$ in CMSi and $5.11 \pm 0.08 \times 10^{-6} \AA^{-2}$ in $\mathrm{H}_{2} \mathrm{O}$. ${ }^{c}$ In $\mathrm{D}_{2} \mathrm{O},-0.092$ $\pm 0.06 \times 10^{-6} \AA^{-2}$ in CM4 $-0.112 \pm 0.04 \times 10^{-6} \AA^{-2}$ in CMSi and $-0.14 \pm 0.03 \times 10^{-6} \AA^{-2}$ in $\mathrm{H}_{2} \mathrm{O}$.

It has been shown experimentally and indicated by computer simulation that AmB binds more strongly to ergosterol than cholesterol [51,52], which has been suggested as the basis of its specificity as an antifungal agent. At the concentration employed in this study ( $1 \mathrm{mM}), \mathrm{AmB}$ is mostly in an aggregated form in solution, and as no AmB aggregates are observed on the membrane surface, it can be assumed that the active form of $\mathrm{AmB}$ is the free molecules that have a concentration up to the 
critical micellization concentration ( $\mathrm{cmc}$ ) of $0.63 \mu \mathrm{M}$ [53]. This is of the order of typical minimum inhibitory concentrations (MIC) values of $\mathrm{AmB}$, which are in the $1 \mu \mathrm{g} / \mathrm{mL}$ range for a wide range of yeast species [54]. It has been proposed that the AmB toxic side effects are related to membrane permeabilization by pore formation, and with cholesterol, this has only been observed at high AmB concentrations [55]. Our data show that there is more significant AmB insertion in cholesterol-containing membranes even without pore formation, which can contribute to some of the toxicity of AmB due to the sequestration of cholesterol within the membranes, lowering its bioavailability.

\section{Discussion}

Previous reports of AmB using neutron scattering from POPC-based model membranes and vesicles $[18,19]$ have suggested that $A m B$ causes the bilayers to become thicker, and similar observations have also been made in DPPC monolayers containing much higher amounts of sterols using X-ray reflection [56]. However, a direct comparison between our bilayer data and monolayer data is not straightforward. Our experiments, designed to detect both the sterols and AmB using selective deuteration, show similar AmB insertion in both POPC and POPC-ergosterol bilayers without any significant changes in the membrane thickness but a decrease in interfacial roughness indicating a small ordering effect. AmB inserts into the cholesterol-containing POPC bilayers to a higher degree, but it is not able to extract cholesterol. In contrast to the pure $\mathrm{d}_{82}$ POPC and $\mathrm{d}_{82}$ POPC-hChol, in which the SLD of both headgroup regions was lowered by AmB, only the outer headgroup SLD decreased in the $\mathrm{d}_{82}$ POPC-hErg bilayers in a manner that suggests it is related to the extraction of ergosterol.

At first glance, the differences observed in sterol extraction seem to be directly related to the stronger binding of AmB to ergosterol than to cholesterol $[4,5,7,8,57]$. However, it is important to note that the insertion of AmB into fungal cells may also be regulated by other properties of cell membranes that are composed of many different lipids. One of these properties is the hydrophobic thickness of the membranes, which depends on the lipid composition. The length of an AmB molecule is $21 \AA$ [57], which means that in POPC-based membranes that have a hydrophobic thickness of $>30 \AA$ and a total thickness of $\approx 50 \AA$, more than one AmB molecule is needed to span the membrane, as illustrated in Figures 1-3, and the slight thickening and reduction in interfacial roughness indicate that accommodating AmB also leads to ordering of the lipids. In contrast, in polyunsaturated P. pastoris yeast phospholipid membranes, which have a lower hydrophobic thickness $(26 \pm 1 \AA)$, the insertion of AmB leads to membrane thinning to $24 \pm 1 \AA$, both in the absence and presence of ergosterol, which suggests that these membranes can be spanned by only one AmB molecule. This indicates that lipid composition can determine the mode of $\mathrm{AmB}$ insertion, but also that $\mathrm{AmB}$ insertion can modify the membrane thickness. A similar effect has been observed previously in saturated bilayers of 1,2-sn-di-myristoyl-phoshatidylcholine (DMPC) and 1,2-sn-di-steraoyl-phosphatidylcholine (DSPC) in which the different chain lengths by ${ }^{13} \mathrm{C}$ NMR spectroscopy [12].

We also could not observe any AmB aggregates or AmB layer outside the POPC or POPC-sterol membranes, as was previously seen for reconstituted P. pastoris extract bilayers [28]. Previous investigations of AmB in lipid monolayers [20,56] also did not detect an AmB layer in contact with the synthetic PC lipids, which suggests that the headgroups of the other lipid classes present in the yeast phospholipids play an important role in the interaction with AmB. The main mode of action of the recently proposed AmB sponge layer was proposed to be ergosterol extraction into the sponge layer, but our results show unambiguously that the extraction can also take place in the absence of a sponge layer attached to the membrane. As opposed to the methods used previously [15] to detect the association of ergosterol with $\mathrm{AmB}$ and removal from POPC, we have detected the ergosterol extraction (and lack of cholesterol extraction) in an in situ experiment under physiologically relevant solution conditions. In the previous NMR-based studies, the full membrane structure was not characterized, and the AmB sponge was observed by electron microscopy of dried samples in vacuum. Therefore, it is possible that no large AmB sponge forms on POPC-based bilayers in situ, even if this is the case for more complex membranes such as those derived from yeast, and therefore, it is important to make 
these structural observations in situ using biologically relevant model membranes. We also did not observe significant water insertion in the samples that could have indicated a systematic pore formation mechanism, and the volume of ergosterol extracted was filled by AmB molecules. This may be due to a transient nature of the pores that occur during AmB insertion and ergosterol extraction, as the presence of amphipathic molecules in membranes can lead to permeabilization without the formation of open pores. On the other hand, the total volume of water inside the AmB channels would be very small and of the order of the detection limits of our NR measurements when the volume fraction of $\mathrm{AmB}$ is of the order of $5 \%$ relative to the lipids.

In our measurements, the concentration of free $\mathrm{AmB}$ is at the $\mathrm{cmc}(0.63 \mu \mathrm{M})$, with the remaining AmB present as aggregates. The experiments were conducted with a single large supported bilayer $(\approx 5.5 \mu \mathrm{g} / 7.2 \mathrm{nmol}$ of lipids) in contact with $1 \mathrm{~mL}$ of AmB solution $(0.63 \mathrm{nmol}$ free AmB), which gives an AmB to lipid ratio of 0.0875 , or 11.4 lipids per AmB. This is of the same order of magnitude as the amount of $\mathrm{AmB}$ we see inserted in the membranes. In our experiments, we also have a much larger reservoir of aggregated $\mathrm{AmB}$ that is able to equilibrate with the membrane upon free AmB insertion, and therefore, it is not possible to directly compare this to known minimum inhibitory concentrations (MIC) of AmB. To our knowledge, the stoichiometry between known MICs and ergosterol extraction/pore formation has not been quantified.

Our results were obtained using AmB in solution; however, our observations are also relevant for its liposomal formulations, which serve to regulate the concentration of free AmB. These formulations, which have been observed to pass the fungal cell wall as intact liposomes [58], and attach to the fungal cell membrane surface, potentially present an additional method of delivery by direct exchange of $\mathrm{AmB}$ (and potentially also lipids) with the target membranes in a manner similar to that detected for phospholipids and cholesterol $[59,60]$, but this has not been experimentally observed to our knowledge.

\section{Conclusions}

We have shown unambiguously that $\mathrm{AmB}$ is able to insert into both pure POPC as well as POPC-sterol bilayers, and that it extracts ergosterol but not cholesterol, although AmB inserts to a higher degree in POPC-cholesterol membranes. Our results also show that the behavior of AmB on these model lipid bilayers is different from that observed on yeast phospholipid extract bilayers that reflect the lipid composition of the fungal target membranes of AmB. Notably, the AmB sponge layer proposed to be responsible for extracting ergosterol as part of the antifungal mechanism does not form in situ on simple model bilayers composed of POPC and the sterols, but ergosterol extraction occurs nevertheless. POPC-based membranes do not undergo significant thickness changes and require two AmB molecules to span their thickness, whereas yeast lipid bilayers that have a lower hydrophobic thickness become thinner so that a single AmB molecule can span their hydrophobic interior. This clearly demonstrates that lipid composition can determine the mode of AmB insertion and subsequent structural changes to the membrane. The pore-formation process, evidenced by membrane permeabilization, has been the accepted model of AmB for several decades, and is the most obvious one. Our results show unambiguously that both AmB insertion and ergosterol extraction occur, and while no aqueous pores were directly detected, the process of AmB insertion and ergosterol extraction can both be the origin of increased membrane permeability. As such, the pore formation and ergosterol extraction models are not mutually exclusive and may be both contributing to the antifungal mechanism of AmB.

Supplementary Materials: The following are available online at http://www.mdpi.com/2079-4991/10/12/2439/s1, Figure S1: Separation by gas chromatography of the different sterols of interest, and mass spectra of ergosterol extracted from P. pastoris grown in either hydrogenous or deuterated conditions, Figure S2: QCM-D traces of 3 successive additions of a 1mM AmB D2O/DMSO 9:1 v/v\% followed by rinsing with D2O/DMSO 9/1 v/v\% at $30^{\circ} \mathrm{C}$. 
Author Contributions: Conceptualization, H.P.W.-K.; methodology, R.D., H.P.W.-K., V.L., M.H.; software, A.N.; formal analysis, R.D., H.P.W.-K.; investigation, R.D., H.P.W.-K., A.N.; resources, H.P.W.-K., G.F., W.K.; writing—original draft preparation, R.D., H.P.W.-K.; writing—review and editing, H.P.W.-K., G.F., W.K.; M.H., A.N., supervision, H.P.W.-K., G.F., W.K.; project administration, H.P.W.-K., W.K.; funding acquisition, H.P.W.-K., G.F., W.K. All authors have read and agreed to the published version of the manuscript.

Funding: This research was funded by Lund University, the Swedish Research Council (VR) grant number 2016-01164 the SINE2020 EC grant no. 64500. R. Delhom's Ph.D. thesis work was funded jointly by the European Spallation Source ERIC and Institut Laue-Langevin.

Acknowledgments: The authors acknowledge the Australian Neutron Scattering Center at ANSTO for the awarded neutron beam time, deuteration and sample preparation support from the Australian National Deuteration Facility, the Partnership for Soft Condensed Matter and the D-lab (Partnership for Structural Biology) at the Institut Laue Langevin, Grenoble, as well as the European Spallation Source ERIC Deuteration and Macromolecular Crystallisation Platform and Lund Protein Production Platform.

Conflicts of Interest: The authors declare no conflict of interest.

\section{References}

1. Gallis, H.A.; Drew, R.H.; Pickard, W.W. Amphotericin B: 30 Years of Clinical Experience. Clin. Infect. Dis. 1990, 12, 308-329. [CrossRef] [PubMed]

2. Hartsel, S.; Bolard, J. Amphotericin B: New life for an old drug. Trends Pharmacol. Sci. 1996, 17, 445-449. [CrossRef]

3. Hamill, R.J. Amphotericin B formulations: A comparative review of efficacy and toxicity. Drugs 2013, 73, 919-934. [CrossRef] [PubMed]

4. Matsumori, N.; Tahara, K.; Yamamoto, H.; Morooka, A.; Doi, M.; Oishi, T.; Murata, M. Direct interaction between amphotericin $\mathrm{B}$ and ergosterol in lipid bilayers as revealed by $2 \mathrm{H}$ NMR spectroscopy. J. Am. Chem. Soc. 2009, 131, 11855-11860. [CrossRef]

5. Gray, K.C.; Palacios, D.S.; Dailey, I.; Endo, M.M.; Uno, B.E.; Wilcock, B.C.; Burke, M.D. Amphotericin primarily kills yeast by simply binding ergosterol. Proc. Natl. Acad. Sci. USA 2012, 109, 2234-2239. [CrossRef]

6. Gabrielska, J.; Gagos, M.; Gubernator, J.; Gruszecki, W.I. Binding of antibiotic amphotericin B to lipid membranes: A 1H NMR study. FEBS Lett. 2006, 580, 2677-2685. [CrossRef]

7. Ciesielski, F.; Griffin, D.C.; Loraine, J.; Rittig, M.; Delves-Broughton, J.; Bonev, B.B. Recognition of Membrane Sterols by Polyene Antifungals Amphotericin B and Natamycin, A 13C MAS NMR Study. Front. Cell Dev. Biol. 2016, 4, 212-253. [CrossRef]

8. Huang, W.; Zhang, Z.; Han, X.; Tang, J.; Wang, J.; Dong, S.; Wang, E. Ion Channel Behavior of Amphotericin B in Sterol-Free and Cholesterol- or Ergosterol-Containing Supported Phosphatidylcholine Bilayer Model Membranes Investigated by Electrochemistry and Spectroscopy. Biophys. J. 2002, 83, 3245-3255. [CrossRef]

9. Baginski, M.; Resat, H.; Borowski, E. Comparative molecular dynamics simulations of amphotericin B-cholesterol/ergosterol membrane channels. Biochim. Biophys. Acta (BBA) Biomembr. 2002, 1567, 63-78. [CrossRef]

10. Venegas, B.; González-Damián, J.; Celis, H.; Ortega-Blake, I. Amphotericin B Channels in the Bacterial Membrane: Role of Sterol and Temperature. Biophys. J. 2003, 85, 2323-2332. [CrossRef]

11. Matsuoka, S.; Murata, M. Membrane permeabilizing activity of amphotericin B is affected by chain length of phosphatidylcholine added as minor constituent. Biochim. Biophys. Acta (BBA) Biomembr. 2003, 1617, 109-115. [CrossRef] [PubMed]

12. Matsuoka, S.; Ikeuchi, H.; Matsumori, N.; Murata, M. Dominant formation of a single-length channel by amphotericin B in dimyristoylphosphatidylcholine membrane evidenced by 13C-31P rotational echo double resonance. Biochemistry 2005, 44, 704-710. [CrossRef] [PubMed]

13. Mouri, R.; Konoki, K.; Matsumori, N.; Oishi, T.; Murata, M. Complex formation of amphotericin B in sterol-containing membranes as evidenced by surface plasmon resonance. Biochemistry 2008, 47, 7807-7815. [CrossRef] [PubMed]

14. Romero, E.A.; Valdivieso, E.; Cohen, B.E. Formation of Two Different Types of Ion Channels by Amphotericin B in Human Erythrocyte Membranes. J. Membr. Biol. 2009, 230, 69-81. [CrossRef] [PubMed] 
15. Anderson, T.M.; Clay, M.C.; Cioffi, A.G.; Diaz, K.A.; Hisao, G.S.; Tuttle, M.D.; Nieuwkoop, A.J.; Comellas, G.; Maryum, N.; Wang, S.; et al. Amphotericin forms an extramembranous and fungicidal sterol sponge. Nat. Chem. Biol. 2014, 10, 400-406. [CrossRef] [PubMed]

16. Dufourc, E.J. Sterols and membrane dynamics. J. Chem. Biol. 2008, 1, 63-77. [CrossRef]

17. Chudzik, B.; Koselski, M.; Czuryło, A.; Trębacz, K.; Gagoś, M. A new look at the antibiotic amphotericin B effect on Candida albicans plasma membrane permeability and cell viability functions. Eur. Biophys. J. 2015, 44, 77-90. [CrossRef]

18. Foglia, F.; Drake, A.F.; Terry, A.E.; Rogers, S.E.; Lawrence, M.J.; Barlow, D.J. Small-angle neutron scattering studies of the effects of amphotericin B on phospholipid and phospholipid-sterol membrane structure. Biochim. Biophys. Acta (BBA) Biomembr. 2011, 1808, 1574-1580. [CrossRef]

19. Foglia, F.; Lawrence, M.J.; Demee, B.; Fragneto, G.; Barlow, D. Neutron diffraction studies of the interaction between amphotericin B and lipid-sterol model membranes. Sci. Rep. 2012, 2, 806-809. [CrossRef]

20. Foglia, F.; Fragneto, G.; Clifton, L.A.; Lawrence, M.J.; Barlow, D.J. Interaction of Amphotericin B with Lipid Monolayers. Langmuir 2014, 30, 9147-9156. [CrossRef]

21. Harayama, T.; Riezman, H. Understanding the diversity of membrane lipid composition. Nat. Rev. Mol. Cell Biol. 2018, 19, 281. [CrossRef] [PubMed]

22. Yepuri, N.R.; Darwish, T.A.; Krause-Heuer, A.M.; Leung, A.E.; Delhom, R.; Wacklin, H.P.; Holden, P.J. Synthesis of Perdeuterated 1-Palmitoyl-2-oleoyl-sn-glycero-3-phosphocholine ([D82]POPC) and Characterisation of Its Lipid Bilayer Membrane Structure by Neutron Reflectometry. ChemPlusChem 2016, 81, 315-321. [CrossRef] [PubMed]

23. Fragneto, G.; Delhom, R.; Joly, L.; Scoppola, E. Neutrons and model membranes: Moving towards complexity. Curr. Opin. Colloid Interface Sci. 2018, 38, 108-121. [CrossRef]

24. Gerelli, Y.; de Ghellinck, A.; Jouhet, J.; Laux, V.; Haertlein, M.; Fragneto, G. Multi-lamellar organization of fully deuterated lipid extracts of yeast membranes. Acta Crystallogr. Sect. D Biol. Crystallogr. 2014, 70, 3167-3176. [CrossRef]

25. Luchini, A.; Delhom, R.; Cristiglio, V.; Knecht, W.; Wacklin-Knecht, H.; Fragneto, G. Effect of ergosterol on the interlamellar spacing of deuterated yeast phospholipid multilayers. Chem. Phys. Lipids 2020, 227, 104873. [CrossRef]

26. Luchini, A.; Delhom, R.; Demé, B.; Laux, V.; Moulin, M.; Haertlein, M.; Pichler, H.; Strohmeier, G.A.; Wacklin, H.; Fragneto, G. The impact of deuteration on natural and synthetic lipids: A neutron diffraction study. Colloids Surf. B Biointerfaces 2018, 168, 126-133. [CrossRef]

27. de Ghellinck, A.; Schaller, H.; Laux, V.; Haertlein, M.; Sferrazza, M.; Maréchal, E.; Wacklin, H.; Jouhet, J.; Fragneto, G. Production and Analysis of Perdeuterated Lipids from Pichia pastoris Cells. PLoS ONE 2014, 9, e92999. [CrossRef]

28. de Ghellinck, A.; Fragneto, G.; Laux, V.; Haertlein, M.; Jouhet, J.; Sferrazza, M.; Wacklin, H. Lipid polyunsaturation determines the extent of membrane structural changes induced by Amphotericin $\mathrm{B}$ in Pichia pastoris yeast. Biochim. Biophys. Acta (BBA) Biomembr. 2015, 1848, 2317-2325. [CrossRef]

29. Wacklin, H.P. Composition and asymmetry in supported membranes formed by vesicle fusion. Langmuir 2011, 27, 7698-7707. [CrossRef]

30. Hayter, J.B.; Highfield, R.R.; Pullman, B.J.; Thomas, R.K.; McMullen, A.I.; Penfold, J. Critical reflection of neutrons. A new technique for investigating interfacial phenomena. J. Chem. Soc. Faraday Trans. 1 Phys. Chem. Condens. Phases 1981, 77, 1437-1448. [CrossRef]

31. Vacklin, H.P.; Tiberg, F.; Fragneto, G.; Thomas, R.K. Composition of Supported Model Membranes Determined by Neutron Reflection. Langmuir 2005, 21, 2827-2837. [CrossRef] [PubMed]

32. Wacklin, H.P.; Bremec, B.B.; Moulin, M.; Rojko, N.; Haertlein, M.; Forsyth, T.; Anderluh, G.; Norton, R.S. Neutron reflection study of the interaction of the eukaryotic pore-forming actinoporin equinatoxin II with lipid membranes reveals intermediate states in pore formation. Biochim. Biophys. Acta (BBA) Biomembr. 2016, 1858, 640-652. [CrossRef] [PubMed]

33. James, M.; Nelson, A.; Holt, S.A.; Saerbeck, T.; Hamilton, W.A.; Klose, F. The multipurpose time-of-flight neutron reflectometer "Platypus" at Australia's OPAL reactor. Nucl. Instrum. Methods Phys. Res. Sect. A Accel. Spectrometers Detect. Assoc. Equip. 2011, 632, 112-123. [CrossRef]

34. Nelson, A. Co-refinement of multiple-contrast neutron/X-ray reflectivity data using MOTOFIT. J. Appl. Crystallogr. 2006, 39, 273-276. [CrossRef] 
35. Born, M.; Wolf, E. Principles of Optics: Electromagnetic Theory of Propagation, Interference and Diffraction of Light, 7th ed.; Cambridge University Press: Cambridge, UK, 1999. [CrossRef]

36. Armen, R.S.; Uitto, O.D.; Feller, S.E. Phospholipid component volumes: Determination and application to bilayer structure calculations. Biophys. J. 1998, 75, 734-744. [CrossRef]

37. Nagle, J.F.; Tristram-Nagle, S. Structure of lipid bilayers. Biochim. Biophys. Acta (BBA) Rev. Biomembr. 2000, 1469, 159-195. [CrossRef]

38. Greenwood, A.I.; Tristram-Nagle, S.; Nagle, J.F. Partial molecular volumes of lipids and cholesterol. Chem. Phys. Lipids 2006, 143, 1-10. [CrossRef]

39. Johnson, S.J.; Bayerl, T.M.; McDermott, D.C.; Adam, G.W.; Rennie, A.R.; Thomas, R.K.; Sackmann, E. Structure of an Adsorbed Dimyristoylphosphatidylcholine Bilayer Measured with Specular Reflection of Neutrons. Biophys. J. 1991, 59, 289-294. [CrossRef]

40. Cremer, P.S.; Boxer, S.G. Formation and spreading of lipid bilayers on planar glass supports. J. Phys. Chem. B 1999, 103, 2554-2559. [CrossRef]

41. Zwang, T.J.; Fletcher, W.R.; Lane, T.J.; Johal, M.S. Quantification of the Layer of Hydration of a Supported Lipid Bilayer. Langmuir 2010, 26, 4598-4601. [CrossRef]

42. Waldie, S.; Lind, T.K.; Browning, K.; Moulin, M.; Haertlein, M.; Forsyth, V.T.; Luchini, A.; Strohmeier, G.A.; Pichler, H.; Maric, S.; et al. Localization of Cholesterol within Supported Lipid Bilayers Made of a Natural Extract of Tailor-Deuterated Phosphatidylcholine. Langmuir 2018, 34, 472-479. [CrossRef]

43. Marquardt, D.; Heberle, F.A.; Greathouse, D.V.; Koeppe, R.E.; Standaert, R.F.; Van Oosten, B.J.; Harroun, T.A.; Kinnun, J.J.; Williams, J.A.; Wassall, S.R.; et al. Lipid bilayer thickness determines cholesterol's location in model membranes. Soft Matter 2016, 12, 9417-9428. [CrossRef] [PubMed]

44. Åkesson, A.; Lind, T.; Ehrlich, N.; Stamou, D.; Wacklin, H.; Cárdenas, M. Composition and structure of mixed phospholipid supported bilayers formed by POPC and DPPC. Soft Matter 2012, 8, 5658-5665. [CrossRef]

45. Soranzo, T.; Martin, D.K.; Lenormand, J.-L.; Watkins, E.B. Coupling neutron reflectivity with cell-free protein synthesis to probe membrane protein structure in supported bilayers. Sci. Rep. 2017, 7, 3399. [CrossRef] [PubMed]

46. Kučerka, N.; Nieh, M.-P.; Katsaras, J. Fluid phase lipid areas and bilayer thicknesses of commonly used phosphatidylcholines as a function of temperature. Biochim. Biophys. Acta (BBA) Biomembr. 2011, 1808, 2761-2771. [CrossRef]

47. Dabkowska, A.P.; Collins, L.E.; Barlow, D.J.; Barker, R.; McLain, S.E.; Lawrence, M.J.; Lorenz, C.D. Modulation of Dipalmitoylphosphatidylcholine Monolayers by Dimethyl Sulfoxide. Langmuir 2014, 30, 8803-8811. [CrossRef]

48. Hung, W.-C.; Lee, M.-T.; Chung, H.; Sun, Y.-T.; Chen, H.; Charron, N.E.; Huang, H.W. Comparative Study of the Condensing Effects of Ergosterol and Cholesterol. Biophys. J. 2016, 110, 2026-2033. [CrossRef]

49. Baran, M.; Borowski, E.; Mazerski, J. Molecular modeling of amphotericin B-ergosterol primary complex in water II. Biophys. Chem. 2009, 141, 162-168. [CrossRef]

50. Urbina, J.A.; Pekerar, S.; Le, H.-B.; Patterson, J.; Montez, B.; Oldfield, E. Molecular order and dynamics of phosphatidylcholine bilayer membranes in the presence of cholesterol, ergosterol and lanosterol: A comparative study using 2H-, 13C- and 31P-NMR spectroscopy. Biochim. Biophys. Acta (BBA) Biomembr. 1995, 1238, 163-176. [CrossRef]

51. Readio, J.D.; Bittman, R. Equilibrium binding of amphotericin B and its methyl ester and borate complex to sterols. Biochim. Biophys. Acta (BBA) Biomembr. 1982, 685, 219-224. [CrossRef]

52. Neumann, A.; Baginski, M.; Czub, J. How Do Sterols Determine the Antifungal Activity of Amphotericin B? Free Energy of Binding between the Drug and Its Membrane Targets. J. Am. Chem. Soc. 2010, 132, 18266-18272. [CrossRef] [PubMed]

53. Tancréde, P.; Barwicz, J.; Jutras, S.; Gruda, I. The effect of surfactants on the aggregation state of amphotericin B. Biochim. Biophys. Acta (BBA) Biomembr. 1990, 1030, 289-295. [CrossRef]

54. Borman, A.M.; Muller, J.; Walsh-Quantick, J.; Szekely, A.; Patterson, Z.; Palmer, M.D.; Fraser, M.; Johnson, E.M. MIC distributions for amphotericin B, fluconazole, itraconazole, voriconazole, flucytosine and anidulafungin and 35 uncommon pathogenic yeast species from the UK determined using the CLSI broth microdilution method. J. Antimicrob. Chemother. 2020, 75, 1194-1205. [CrossRef] [PubMed]

55. Barwicz, J.; Tancrède, P. The effect of aggregation state of amphotericin-B on its interactions with cholesterolor ergosterol-containing phosphatidylcholine monolayers. Chem. Phys. Lipids 1997, 85, 145-155. [CrossRef] 
56. Kamiński, D.M.; Czernel, G.; Murphy, B.; Runge, B.; Magnussen, O.M.; Gagoś, M. Effect of cholesterol and ergosterol on the antibiotic amphotericin $B$ interactions with dipalmitoylphosphatidylcholine monolayers: X-ray reflectivity study. Biochim. Biophys. Acta (BBA) Biomembr. 2014, 1838, 2947-2953. [CrossRef]

57. Van Hoogevest, P.; De Kruijff, B. Effect of amphotericin B on cholesterol-containing liposomes of egg phosphatidylcholine and didocosenoyl phosphatidylcholine. A refinement of the model for the formation of pores by amphotericin B in membranes. Biochim. Biophys. Acta (BBA) Biomembr. 1978, 511, 397-407. [CrossRef]

58. Walker, L.; Sood, P.; Lenardon, M.D.; Milne, G.; Olson, J.; Jensen, G.; Wolf, J.; Casadevall, A.; Adler-Moore, J.; Gow, N.A.R. The Viscoelastic Properties of the Fungal Cell Wall Allow Traffic of AmBisome as Intact Liposome Vesicles. mBio 2018, 9, e02383-17. [CrossRef]

59. Gerelli, Y.; Porcar, L.; Lombardi, L.; Fragneto, G. Lipid Exchange and Flip-Flop in Solid Supported Bilayers. Langmuir 2013, 29, 12762-12769. [CrossRef]

60. Garg, S.; Porcar, L.; Woodka, A.C.; Butler, P.D.; Perez-Salas, U. Noninvasive Neutron Scattering Measurements Reveal Slower Cholesterol Transport in Model Lipid Membranes. Biophys. J. 2011, 101, 370-377. [CrossRef]

Publisher's Note: MDPI stays neutral with regard to jurisdictional claims in published maps and institutional affiliations.

(C) 2020 by the authors. Licensee MDPI, Basel, Switzerland. This article is an open access article distributed under the terms and conditions of the Creative Commons Attribution (CC BY) license (http://creativecommons.org/licenses/by/4.0/). 